\title{
Axillary Response in Patients Undergoing Neoadjuvant Endocrine Treatment for Node-Positive Breast Cancer: Systematic Literature Review and NCDB Analysis
}

\author{
Arielle Stafford, MD', Austin Williams, $\mathbf{M D}^{2}$, Kirsten Edmiston, $\mathbf{M D}^{1}$, Costanza Cocilovo, $\mathbf{M D}^{1}$, \\ Robert Cohen, MD ${ }^{1}$, Sara Bruce, MD ${ }^{1}$, Kahyun Yoon-Flannery, DO, MPH ${ }^{3,4}$, and Lucy De La Cruz, MD ${ }^{1}$ \\ ${ }^{1}$ Division of Breast Surgery, Department of Surgery, Inova Health System, Schar Cancer Institute, Fairfax, VA; \\ ${ }^{2}$ Department of Surgery, Lankenau Medical Center, Wynnewood, PA; ${ }^{3}$ Comprehensive Breast Center, Jefferson Health \\ New Jersey, Sewell, NJ; ${ }^{4}$ Department of Surgery, Rowan SOM, Stratford, NJ
}

\begin{abstract}
Background. Several studies have proven that neoadjuvant endocrine therapy (NET) has a similar beneficial therapeutic effect in estrogen-positive $\left(\mathrm{ER}^{+}\right)$breast cancer (BC) with improved breast conservation rate in patients undergoing NET versus neoadjuvant chemotherapy (NAC). The impact of axillary complete pathologic response (pCR) is less clear. We evaluate the impact of NET on axillary downstaging and surgical management.

Methods. Using the National Cancer Database (NCDB), we identified all patients with node positive $\left(\mathrm{N}^{+}\right), \mathrm{ER}^{+}$, HER $^{2-}$ BC undergoing NET and performed a systemic review of literature using PRISMA guidelines.

Results. The literature review identified 1479 clinically $\mathrm{N}^{+}$patients in four studies, 148 of whom had axillary pCR $(10.0 \%)$. In the two studies of patients with invasive lobular carcinoma (ILC), $7.8 \%(69 / 883)$ of clinically $\mathrm{N}^{+}$ patients had axillary pCR. The NCDB query identified 4580 female patients with clinically $\mathrm{N}^{+} \mathrm{ER}^{+} \mathrm{HER}^{2-} \mathrm{BC}$
\end{abstract}

Kahyun Yoon-Flannery and Lucy M. De La Cruz were co-senior authors of this work.

Electronic supplementary material The online version of this article (https://doi.org/10.1245/s10434-020-08905-9) contains supplementary material, which is available to authorized users.

(C) Society of Surgical Oncology 2020

First Received: 14 May 2020

Accepted: 8 July 2020;

Published Online: 9 September 2020

L. De La Cruz, MD

e-mail: Lucy.delacruz@inova.org who underwent NET from 2010 to 2016 with mean age of 61.4 years. Patients who achieved a pCR were more likely to have N1 disease ( $p$ 0.008), moderately differentiated tumors ( $p 0.003)$, and ductal histology ( $p 0.04)$. There was no statistically significant difference in race, comorbidity score, education, income, hospital setting, or clinical tumor stage. Of the 4580 total patients, 663 (14.48\%) had an axillary pCR (pN0) after NET, and 3917 (85.52\%) remained $\mathrm{pN}+$.

Conclusions. We found that patients who underwent NET for $\mathrm{N}^{+}$disease had a higher axillary pCR than previously reported (10\%) in smaller studies. Although NET is not a common treatment option for women with $\mathrm{N}^{+} \mathrm{ER}^{+} \mathrm{HER}^{2-}$ $\mathrm{BC}$, it may be a suitable option for axillary downstaging, which is currently underutilized.

In 2019, approximately 268,600 new cases of invasive breast cancer (IBC) and 48,100 cases of ductal carcinoma in situ (DCIS) were diagnosed among U.S. women, and more than $80 \%$ of breast cancers (BC) were diagnosed among women aged $\geq 50$ years. ${ }^{1}$ Hormone receptor-positive $\left(\mathrm{HR}^{+}\right)$/human epidermal growth factor receptor 2 (HER2)-negative BC is by far the most common subtype. ${ }^{1}$ In patients with $\mathrm{HR}^{+}$breast cancer, the innate exposure to endogenous estrogen results in dimerization of the estrogen receptor (ER) promoting estrogen gene transcription. ${ }^{2}$ Multiple endocrine therapies have been developed for adjuvant use, such as selective ER downregulators, aromatase inhibitors (AIs), and luteinizing hormone releasing agonists. ${ }^{3}$ The role of neoadjuvant endocrine therapy (NET) in the setting of $\mathrm{HR}^{+}$breast cancer in the US is underutilized, typically being reserved for elderly patients, 
who are deemed not suitable for chemotherapy and/or surgery. Neoadjuvant systemic therapy was initially aimed at downstaging inoperable disease but has expanded to diminish disease burden and allow for breast conservation therapy (BCT) and avoidance of axillary node dissection (ALND) in patients who are initially node positive $\left(\mathrm{N}^{+}\right){ }^{4-6}$ It has been previously established that NET can be used in postmenopausal women with strongly $\mathrm{HR}^{+}$breast cancer to achieve downstaging from mastectomy to BCT..$^{4-6}$ While there have been multiple studies demonstrating downstaging of the breast, there is only limited data about the effect of NET on axillary complete pathologic response (pCR) and downstaging in patients with clinically $\mathrm{N}^{+}$ disease.

We aimed to evaluate the impact of NET on axillary downstaging and surgical management, as well as the rates of axillary pCR based on tumor characteristics. This topic is especially pertinent during the current healthcare crisis due to the COVID-19 pandemic, because many patients are being treated with neoadjuvant therapy until hospitals are able to resume normal operating capacity.

\section{METHODS}

A literature search was conducted through the MEDLINE database using PubMed using Preferred Reporting Items for Systematic Reviews and Meta-Analyses (PRISMA) guidelines. Our search terms included "neoadjuvant therapy" [All Fields] AND ("breast" [MeSH Terms] OR "breast" [All Fields]) AND ("surgery" [Subheading] OR "surgery" [All Fields] OR "surgical procedures, operative" [MeSH Terms] OR ("surgical" [All Fields] AND "procedures" [All Fields] AND "operative" [All Fields]) OR "neoadjuvant therapy" [All Fields] OR "surgery" [All Fields] OR "general surgery" [MeSH Terms] OR ("general" [All Fields] AND "surgery" [All Fields]) OR "general surgery" [All Fields]). We filtered all articles from 1988 to 2020 , selecting those containing the key terms: neoadjuvant endocrine therapy; neoadjuvant systemic therapy; neoadjuvant tamoxifen; neoadjuvant aromatase inhibitor; neoadjuvant letrozole; breast cancer; invasive breast cancer; invasive lobular carcinoma; invasive ductal carcinoma; lymph node; node positive; node.

Inclusion criteria were prospective or retrospective clinical trials involving neoadjuvant endocrine therapy that reported axillary response in clinically node-positive patients. Studies needed to include both clinical and pathologic nodal stage. Studies that included both clinical and pathologic nodal stage but did not specifically report axillary response rate in clinically $\mathrm{N}^{+}$patients were excluded.
A manual search of bibliographies of relevant articles was performed based on the PRISMA guidelines. A search of PubMed, including MEDLINE, was performed in August 2019 and was repeated in April 2020 to identify any additional publications.

We then performed a retrospective analysis of female breast cancer patients using the NCDB from 2010 to 2016. The NCDB is a joint American College of Surgeons and American Cancer Society project, where patient-level data is collected from all cancer patients seen at Commission on Cancer (COC) sites. The NCDB captures data from $70 \%$ of all newly diagnosed cancer patients in the United States. ${ }^{11}$

Using the NCDB, we identified all female patients with $\mathrm{N}^{+}$(clinically N1-3), ER-positive $\left(\mathrm{ER}^{+}\right.$), human epidermal growth factor negative $\left(\mathrm{HER}^{2-}\right)$ breast cancer undergoing NET. Progesterone receptor status was not assessed. We identified patients undergoing NET by selecting patients whose initiation of endocrine therapy was greater than 30 days before their first surgical procedure. We did not exclude any patients based on age or presence of metastatic disease. Patients who received neoadjuvant chemotherapy (NAC) were excluded. We stratified patients by nodal response, by pathological node negative (pN0) versus pathological $\mathrm{N}^{+}(\mathrm{pN}+)$, and tumor histology, and compared demographics, cancer characteristics, and treatment information between groups over the study time period. The Charlson-Deyo comorbidity index (CDCI) measured patient medical comorbidities. Tumors were classified by histology based on international classification of disease for oncology. We then performed a comparison analysis of $\mathrm{N}^{+}, \mathrm{ER}^{+}, \mathrm{HER}^{2-}$ undergoing NAC using the same parameters.

All statistical analyses were carried out with SPSS statistical software (IBM Corp., Armonk, NY, USA). Comparisons of patient demographic data were performed using independent $t$ tests, one-way ANOVA, and Chi square tests.

\section{RESULTS}

A total of 509 potential studies were identified by the systematic search, and 10 were identified through other sources. Studies that were duplicated $(n=33)$ were excluded, and the abstracts for the remaining 486 studies were reviewed (Fig. 1). Of these, a total of 148 were selected for full-text review. Nine articles were literature reviews or expert opinions, 48 articles were not related to NET or involved only neoadjuvant chemotherapy (NAC), and 10 did not differentiate between nodal response in NET and NAC. Of the articles involving NET, 26 did not report nodal response, 41 included only clinical or pathologic 
FIG. 1 PRISMA flow chart of the literature

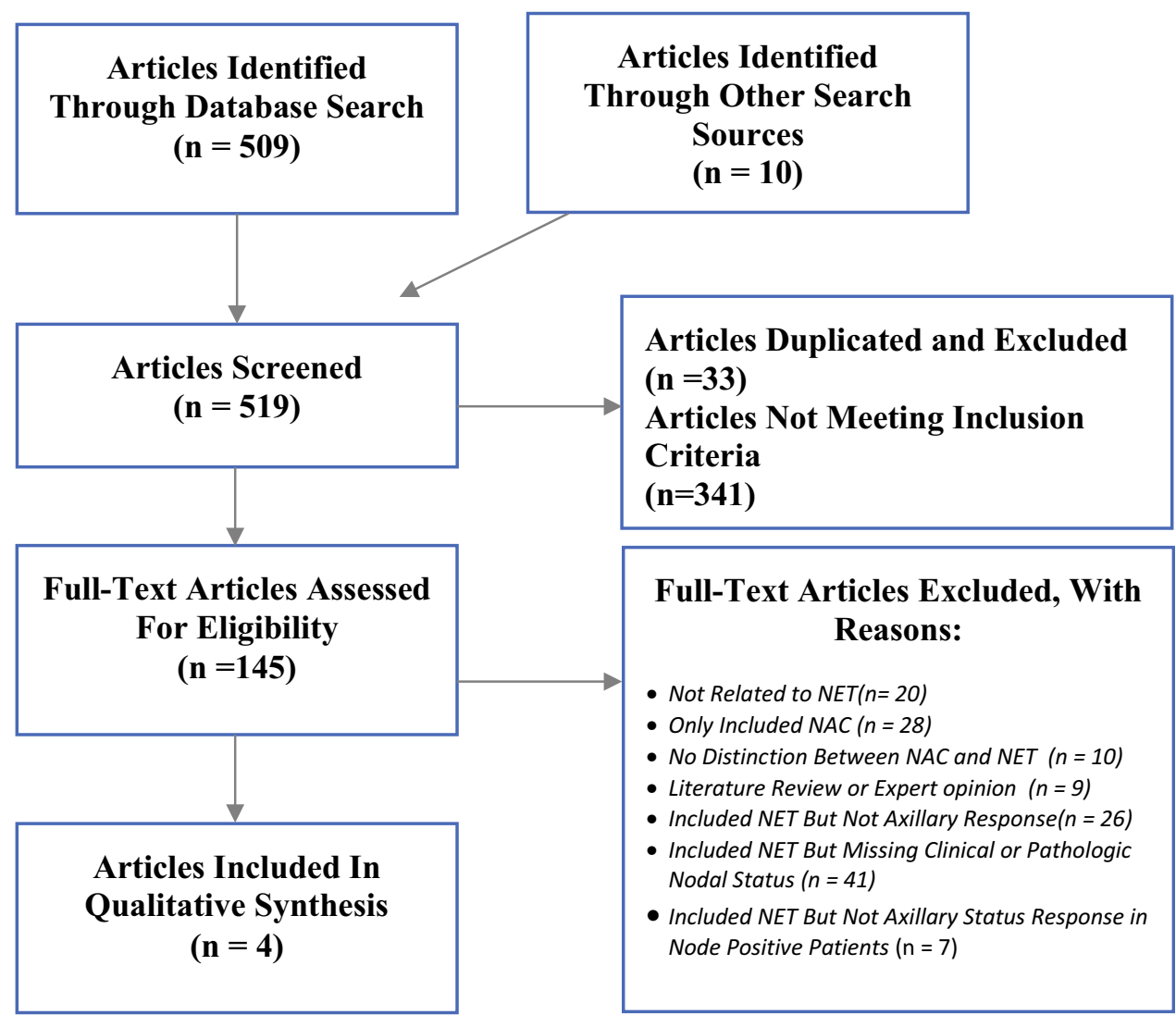

nodal status, and 7 reported both clinical and pathologic nodal status but did not specifically report specific nodal response in clinically $\mathrm{N}^{+}$patients.

A total of four studies met our inclusion criteria and were included for review (Table 1). A 2014 randomized control trial involving postmenopausal women with histologically confirmed strongly $\mathrm{ER}^{+}$( $\geq 50 \%$ on core biopsy) adenocarcinoma (stages I-III) greater than $2 \mathrm{~cm}$ found a $12 \%$ rate of pathologic complete axillary response in clinically $\mathrm{N}^{+}$patients after 6 months of neoadjuvant Exemestane therapy. ${ }^{7}$ This was similar to the findings in a National Cancer Database (NCDB) review from 2012 to 2015, which included clinical stage 2 or 3 (T1-4 and/or N01) $\mathrm{HR}^{+}, \mathrm{HER} 2-\mathrm{IBC}$, and found a $13.3 \%$ rate of $\mathrm{pCR}$ in the axilla after NET. $^{8}$ The median duration of NET was 152 days (interquartile range 97-205 days). Two studies looked specifically at invasive lobular carcinoma (ILC). The first was a prospective clinical trial from 2011 that included postmenopausal women with ER rich (Allred score of $\geq 5$ ) ILC that was too large for BCS or was locally advanced (T4 or N2) undergoing at least 3 months of neoadjuvant Letrozole therapy. ${ }^{9}$ The median length of treatment was 9 months. No patients with clinically $\mathrm{N}^{+}$ had a pCR in the axilla. The second, a 2019 NCDB review of patients with cT1-4c, $\mathrm{LN}^{+}(\mathrm{cN} 1-3), \mathrm{HR}^{+}$ILC from 2004 to 2014 , found an $8.1 \%$ rate of axillary pCR after
NET, but also found a $35.6 \%$ rate of axillary upstaging. ${ }^{10}$ Duration of treatment was not reported. There were 1479 clinically $\mathrm{N}^{+}$patients included in the four studies, 148 of whom had pCR in the axilla (10.0\%). In the two studies of patients with ILC, $7.8 \%(69 / 883)$ of clinically $\mathrm{N}^{+}$patients had pCR in the axilla.

In our NCDB analysis, We identified 4580 female patients with clinically $\mathrm{N}^{+}, \mathrm{ER}^{+}, \mathrm{HER}^{2-}$ breast cancer who underwent NET in the NCDB from 2010 to 2016. The average age of patients included in the study was 61.4 years old. Baseline characteristics are shown in (Table 2). Of these patients, $663(14.48 \%)$ patients had a pCR in the axilla (pN0) after NET, and $3917(85.52 \%)$ remained $\mathrm{pN}^{+}$.

Patients who achieved a pN0 were more likely to have $\mathrm{N} 1$ disease ( $p$ 0.008), moderately differentiated tumors ( $p$ 0.003), and ductal histology ( $p$ 0.04). There was no statistically significant difference in race, comorbidity score, education, income, hospital setting, or clinical tumor stage between patients who achieved pNO and patients who remained $\mathrm{pN}^{+}$. When assessing at percentage of conversion to $\mathrm{pN} 0$ by clinical nodal status, $15.39 \%$ of patients with $\mathrm{cN} 1,11.38 \%$ of patients with clinically N2, and $12 \%$ of patients with clinically $\mathrm{N} 3$ disease had a complete axillary response (Table 3). When stratifying by tumor grade, poorly differentiated tumors had the highest percentage of 


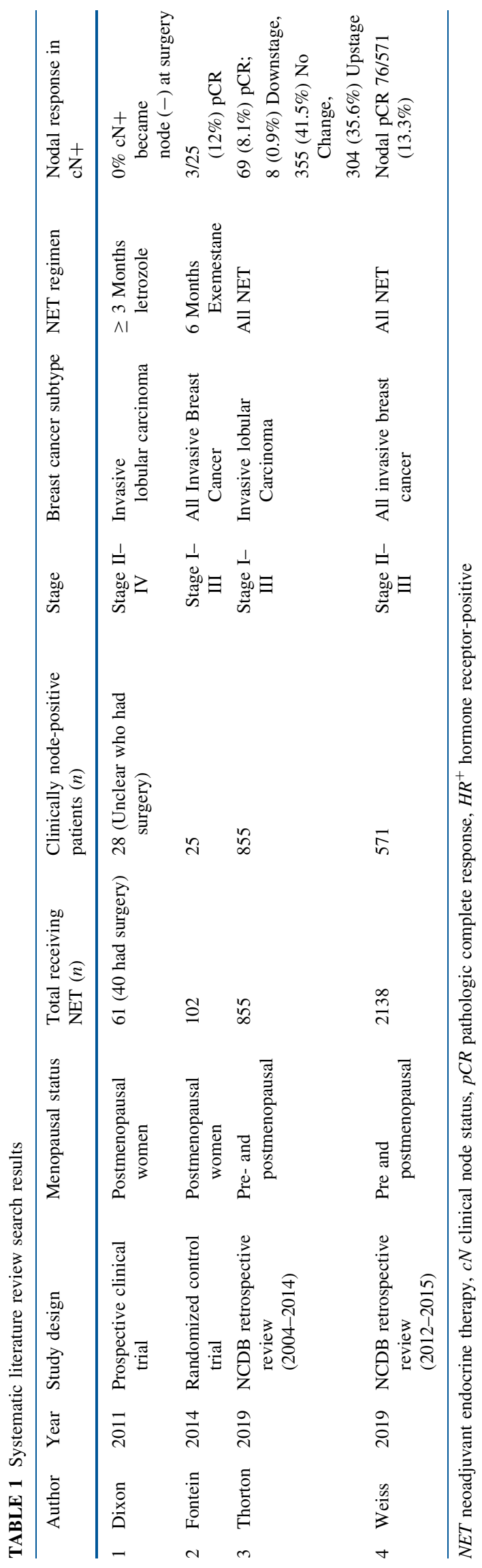

conversion to $\mathrm{pN} 0$ (17.3\%), followed by well-differentiated (14.68\%), and moderately differentiated $(13.08 \%)$. Ductal carcinomas had a significantly higher percentage of axillary complete response than lobular carcinomas $(15.26 \%$ vs. $12.4 \%, p 0.04$ ), whereas $13.01 \%$ of patients with histology classified as other or unknown achieved pNO. Of the 4580 patients included in the study, $974(21.27 \%)$ achieved axillary downstaging, $2199(48.01 \%)$ had no change in nodal status, and $1407(30.72 \%)$ had axillary upstaging (Table 4). When comparing lobular and ductal histology, patients with lobular histology had a lower percentage of axillary downstaging $(15.82 \%$ vs. $22.81 \%)$ and a higher percentage of axillary up-staging versus patients with ductal histology $(40.73 \%$ vs. $27.4 \%)$. Patients who achieved pCR in the axilla were less likely to undergo mastectomy $(67.3 \%$ vs. $75.6 \%, p<0.001)$ and ALND $(57.1 \%$ vs. $87.5 \%, p<0.001)$ than patients who did not achieve pN0. Surgical interventions for both the breast and axilla are shown in Table 5.

In our comparison analysis, we found 25,592 female patients with clinically $\mathrm{N}^{+}, \mathrm{ER}^{+}, \mathrm{HER}^{2-}$ breast cancer who underwent NAC in the NCDB from 2010 to 2016 (Supplementary Table 1). Of these patients, 5361 (20.95\%) achieved pCR in the axilla, and 20,231 (79.05\%) remained $\mathrm{pN}^{+}$after NAC (Supplementary Table 2). When comparing tumor histology, axillary pCR was achieved in $22.87 \%$ of patients with ductal histology and $12.60 \%$ of patients with lobular histology; $28.20 \%$ of all patients achieved axillary downstaging, $30.50 \%$ in ductal carcinoma, and $18.17 \%$ in lobular carcinoma, and $27.31 \%$ had axillary upstaging on pathology, $24.55 \%$ in ductal cancer, and $39.88 \%$ in lobular (Supplementary Table 3). Patients who achieved pCR in the axilla were less likely to undergo mastectomy $(65.8 \%$ vs. $76.1 \%, p<0.001)$ and ALND (64.4\% vs. $89.3 \%, p<0.001)$ than patients who did not achieve pN0 (Supplementary Table 4).

\section{DISCUSSION}

The use of NAC has been primarily for reducing tumor size allowing for BCT in patients who otherwise would not be candidates, as well as for minimizing axillary disease burden therefore decreasing the need for axillary node dissection. Despite the favorable long-term outcome of $\mathrm{HR}^{+}$HER2-negative breast cancer, studies have shown that $\mathrm{HR}^{+}$HER2-negative breast cancer is relatively resistant to NAC compared with HER2-positive and triplenegative cancers. ${ }^{12}$ Endocrine therapy has played a key role in patients with $\mathrm{HR}^{+}$breast cancer in the adjuvant and metastatic setting; however, emphasis on its function in the 
TABLE 2 Axillary response in women with node positive breast cancer undergoing neoadjuvant endocrine therapy

\begin{tabular}{|c|c|c|c|c|}
\hline \multirow[t]{2}{*}{ Baseline characteristics } & \multirow[t]{2}{*}{ All patients } & \multicolumn{3}{|c|}{ Postneoadjuvant axillary status } \\
\hline & & pNO & $\mathrm{pN}+$ & $p$ \\
\hline$n$ & 4580 & 663 & 3917 & \\
\hline Age [years $( \pm$ SD)] & $61.4 \pm 13.8$ & $59.6 \pm 14.2$ & $61.8 \pm 13.6$ & $<0.001$ \\
\hline \multicolumn{5}{|l|}{ Race $(\mathrm{N}, \%)$} \\
\hline White & $3643(79.5)$ & $514(77.5)$ & $3129(79.9)$ & \multirow[t]{4}{*}{0.21} \\
\hline Black & $671(14.7)$ & $112(16.9)$ & $559(14.3)$ & \\
\hline Other & $236(5.2)$ & $36(5.4)$ & $200(5.1)$ & \\
\hline Unknown & $30(0.7)$ & $1(0.2)$ & $29(0.7)$ & \\
\hline \multicolumn{5}{|l|}{ Comorbidity score (N, \%) } \\
\hline 0 & $3682(80.4)$ & $548(82.7)$ & $3134(80.0)$ & \multirow[t]{4}{*}{0.23} \\
\hline 1 & $670(14.6)$ & $87(13.1)$ & $583(14.9)$ & \\
\hline 2 & $171(3.7)$ & $18(2.7)$ & $153(3.9)$ & \\
\hline $3+$ & $57(1.2)$ & $10(1.5)$ & $47(1.2)$ & \\
\hline \multicolumn{5}{|l|}{ Median income $(\mathrm{N}, \%)$} \\
\hline$<\$ 38,000$ & $795(17.4)$ & $123(18.6)$ & $672(17.2)$ & \multirow[t]{5}{*}{0.71} \\
\hline$\$ 38,000-\$ 47,999$ & $1065(23.3)$ & $146(22.0)$ & $919(23.5)$ & \\
\hline$\$ 48,000-\$ 62,999$ & $1221(26.7)$ & $182(27.5)$ & $1039(26.5)$ & \\
\hline$\$ 63,000+$ & $1491(32.6)$ & $212(32.0)$ & $1279(32.7)$ & \\
\hline Unknown & $8(0.2)$ & $0(0.0)$ & $8(0.2)$ & \\
\hline \multicolumn{5}{|c|}{ Percent with no high school diploma $(\mathrm{N}, \%)$} \\
\hline$\geq 21 \%$ & $810(17.7)$ & $137(20.7)$ & $673(17.2)$ & \multirow[t]{5}{*}{0.12} \\
\hline $13.0-20.9 \%$ & $1164(25.4)$ & $154(23.2)$ & $1010(25.8)$ & \\
\hline $7.0-12.9 \%$ & $1486(32.4)$ & $219(33.0)$ & $1267(32.3)$ & \\
\hline$<7.0 \%$ & $1113(24.3)$ & $153(23.1)$ & $960(24.5)$ & \\
\hline Unknown & $7(0.2)$ & $0(0.0)$ & $7(0.2)$ & \\
\hline \multicolumn{5}{|l|}{ Hospital category $(\mathrm{N}, \%)$} \\
\hline Community & $409(8.9)$ & $52(7.8)$ & $357(9.1)$ & \multirow[t]{4}{*}{0.38} \\
\hline Comprehensive community & $1749(38.2)$ & $232(35.0)$ & $1517(38.7)$ & \\
\hline Academic & 1445 (31.6) & $213(32.1)$ & $1232(31.5)$ & \\
\hline National Cancer Institute & $668(14.6)$ & $103(15.5)$ & $565(14.4)$ & \\
\hline \multicolumn{5}{|l|}{ Clinical tumor stage $(\mathrm{N}, \%)$} \\
\hline $\mathrm{T} 1$ & $558(12.2)$ & $83(12.5)$ & $475(12.1)$ & \multirow[t]{5}{*}{0.96} \\
\hline $\mathrm{T} 2$ & $1826(39.9)$ & $272(41.0)$ & $1554(39.7)$ & \\
\hline T3 & $1018(22.2)$ & $148(22.3)$ & $870(22.2)$ & \\
\hline $\mathrm{T} 4$ & $1085(23.7)$ & $154(23.2)$ & $931(23.8)$ & \\
\hline Unknown & $93(2.0)$ & $6(0.9)$ & $87(2.2)$ & \\
\hline \multicolumn{5}{|l|}{ Clinical nodal stage $(\mathrm{N}, \%)$} \\
\hline $\mathrm{N} 1$ & 3477 (75.9) & $535(80.7)$ & $2942(75.1)$ & \multirow[t]{3}{*}{0.008} \\
\hline $\mathrm{N} 2$ & $703(15.3)$ & $80(12.1)$ & $623(15.9)$ & \\
\hline N3 & $400(8.7)$ & $48(7.2)$ & $352(9.0)$ & \\
\hline \multicolumn{5}{|l|}{ Tumor grade $(\mathrm{N}, \%)$} \\
\hline Well-differentiated & $620(13.5)$ & $91(13.7)$ & $529(13.5)$ & \multirow[t]{4}{*}{0.003} \\
\hline Moderately differentiated & $2302(50.3)$ & $301(45.4)$ & $2001(51.1)$ & \\
\hline Poorly differentiated & $1289(28.1)$ & $223(33.6)$ & $1066(27.2)$ & \\
\hline Unknown & $369(8.1)$ & $48(7.2)$ & $321(8.2)$ & \\
\hline
\end{tabular}


TABLE 2 continued

\begin{tabular}{lllll}
\hline Baseline characteristics & All patients & \multicolumn{3}{l}{ Postneoadjuvant axillary status } \\
\cline { 3 - 4 } & & $\mathrm{pN0}$ & $\mathrm{pN}+$ & $p$ \\
\hline Tumor histology $(\mathrm{N}, \%)$ & $3113(68.0)$ & $475(71.6)$ & $2638(67.3)$ & 0.04 \\
Ductal & $847(18.5)$ & $105(15.8)$ & $742(18.9)$ & \\
Lobular & $620(13.5)$ & $83(12.5)$ & $537(13.7)$ & \\
Other/unknown &
\end{tabular}

$p N O$ pathologically node-negative, $p N+$ pathologically node-positive, $N$ node

TABLE 3 Axillary response in women with node positive breast cancer on neoadjuvant endocrine therapy stratified by cancer characteristics

\begin{tabular}{|c|c|c|c|c|c|c|}
\hline \multirow[t]{2}{*}{ Overall } & \multirow[t]{2}{*}{ Total } & \multicolumn{5}{|c|}{ Post-net axillary status } \\
\hline & & pNO & $\%$ of total & $\mathrm{pN}+$ & $\%$ of total & $p$ \\
\hline$n$ & 4580 & 663 & 14.48 & 3917 & 85.52 & \\
\hline \multicolumn{7}{|l|}{ Clinical tumor stage } \\
\hline $\mathrm{T} 1$ & 558 & 83 & 14.87 & 475 & 85.13 & \multirow[t]{5}{*}{0.96} \\
\hline $\mathrm{T} 2$ & 1826 & 272 & 14.90 & 1554 & 85.10 & \\
\hline $\mathrm{T} 3$ & 1018 & 148 & 14.54 & 870 & 85.46 & \\
\hline $\mathrm{T} 4$ & 1085 & 154 & 14.19 & 931 & 85.81 & \\
\hline Unknown & 93 & 6 & 6.45 & 87 & 93.55 & \\
\hline \multicolumn{7}{|l|}{ Clinical nodal stage } \\
\hline N1 & 3477 & 535 & 15.39 & 2942 & 84.61 & \multirow[t]{3}{*}{0.008} \\
\hline $\mathrm{N} 2$ & 703 & 80 & 11.38 & 623 & 88.62 & \\
\hline N3 & 400 & 48 & 12.00 & 352 & 88.00 & \\
\hline \multicolumn{7}{|l|}{ Tumor grade } \\
\hline Well-differentiated & 620 & 91 & 14.68 & 529 & 85.32 & \multirow[t]{4}{*}{0.003} \\
\hline Moderately differentiated & 2302 & 301 & 13.08 & 2001 & 86.92 & \\
\hline Poorly differentiated & 1289 & 223 & 17.30 & 1066 & 82.70 & \\
\hline Unknown & 369 & 48 & 13.01 & 321 & 86.99 & \\
\hline \multicolumn{7}{|l|}{ Tumor histology } \\
\hline Ductal & 3113 & 475 & 15.26 & 2638 & 84.74 & \multirow[t]{3}{*}{0.04} \\
\hline Lobular & 847 & 105 & 12.40 & 742 & 87.60 & \\
\hline Other/unknown & 620 & 83 & 13.39 & 537 & 86.61 & \\
\hline
\end{tabular}

$p N O$ pathologically node-negative; $p N+$ pathologically node-positive; $N$ node
NET setting seems to be gaining momentum. Our study not only evaluated the body of current literature in a systematic fashion; it also elucidated the data from the NCDB.

Recent studies have demonstrated that NET is potentially equally effective in downsizing breast tumors and allowing BCT without the added toxicity from NAC. ${ }^{12-14}$ There are multiple, prospective studies underway to better define the impact of NET on early-stage breast cancer, such as the Alternate Approaches for Clinical Stage II or III Estrogen Receptor-Positive Breast Cancer Neoadjuvant Treatment (ALTERNATE) in Postmenopausal Women: A Phase III Study, which aims to determine pCR as well as other outcomes in this population after treatment with AIs or the combination agents. ${ }^{15}$ In our study, we found that the success of NET is less frequently reported in the literature and that nodal response is not looked at as a main outcome, as it has in most studies evaluating the effect of NAC in the axilla. ${ }^{7-10,16-18}$ In both our literature review and in our NCDB data analysis, we found that there is a significant underutilization of NET despite having an acceptable pCR compared with those patients who undergo NAC for $\mathrm{N}^{+}$disease: $14.5 \%$ in NET versus $20.95 \%$ in NAC. In patients who underwent NET, pCR in the axilla was seen more commonly in those with poorly differentiated tumor characteristics but with lower pretreatment nodal status. We also found that clinical tumor staging did not have a 
TABLE 4 Rates of axillary upstaging and downstaging in women with node-positive breast cancer undergoing neoadjuvant endocrine therapy

\begin{tabular}{|c|c|c|c|c|}
\hline & \multicolumn{3}{|c|}{ Clinical nodal stage } & \multirow[t]{2}{*}{ Total } \\
\hline & $N 1$ & $N 2$ & $N 3$ & \\
\hline \multicolumn{5}{|l|}{ Overall } \\
\hline Total & 3477 & 703 & 400 & 4580 \\
\hline No & 535 & 80 & 48 & 663 \\
\hline N1 & 1656 & 146 & 75 & 1877 \\
\hline $\mathrm{N} 2$ & 852 & 356 & 90 & 1298 \\
\hline $\mathrm{N} 3$ & 434 & 121 & 187 & 742 \\
\hline Downstage & 535 (15.39) & $226(32.15)$ & $213(53.25)$ & 974 (21.27) \\
\hline No change & $1656(47.63)$ & $356(50.64)$ & $187(46.75)$ & $2199(48.01)$ \\
\hline Upstage & $1286(36.99)$ & $121(17.21)$ & 0 & $1407(30.72)$ \\
\hline \multicolumn{5}{|l|}{ Ductal } \\
\hline Total & 2356 & 483 & 274 & 3113 \\
\hline No & 378 & 58 & 39 & 475 \\
\hline N1 & 1193 & 110 & 56 & 1359 \\
\hline $\mathrm{N} 2$ & 572 & 247 & 69 & 888 \\
\hline N3 & 213 & 68 & 110 & 391 \\
\hline Downstage & $378(16.04)$ & 168 (34.78) & $164(59.85)$ & $710(22.81)$ \\
\hline No change & $1193(50.64)$ & 247 (51.14) & $110(40.15)$ & $1550(49.79)$ \\
\hline Upstage & 785 (33.32) & $68(14.08)$ & 0 & $853(27.40)$ \\
\hline \multicolumn{5}{|l|}{ Lobular } \\
\hline Total & 639 & 141 & 67 & 847 \\
\hline No & 84 & 17 & 4 & 105 \\
\hline N1 & 245 & 17 & 3 & 265 \\
\hline N2 & 158 & 72 & 9 & 239 \\
\hline N3 & 152 & 35 & 51 & 238 \\
\hline Downstage & $84(13.15)$ & $34(24.11)$ & $16(23.88)$ & 134 (15.82) \\
\hline No change & $245(38.34)$ & $72(51.06)$ & $51(76.12)$ & $368(43.45)$ \\
\hline Upstage & $310(48.51)$ & $35(24.82)$ & 0 & $345(40.73)$ \\
\hline
\end{tabular}

statistically significant impact on the final pCR, but as expected, ductal carcinomas had a significantly higher percentage of axillary pCR compared with lobular histology. Interestingly enough, NAC is first line for patients who have nodal disease despite low pCR. When we looked at $\mathrm{pCR}$ in the axilla from our systematic review of patients undergoing NET, the reported pCR among studies ranged from 0 to $13.3 \%$, and one study reported no change in nodal status in $41.5 \%$ of patients. Our NCBD analysis found that the $\mathrm{pCR}$ rate was higher than those reported in previous studies, with $14.5 \%$ of all $\mathrm{N}^{+}$patients becoming pN0 after NET. A recent study showed that low pCR rate in patients with luminal-type breast cancer did not consistently result in lower BCS conversion and tumor response rates after NAC compared with those in patients with other breast cancer subtypes. ${ }^{19}$ However, avoiding ALND is not a patient driven surgical decision but a pathologically driven decision if patients have an axillary pCR on final pathology. This can potentially avoid patients the unnecessary morbidity of an ALND.

There are several strengths to this study, including a large, nationwide sample with sufficiently complete data on $\mathrm{HR}^{+} \mathrm{HER}^{2-} \mathrm{N}^{+}$breast cancer patients for the years of analysis and refined subgroup analysis. The study also has several limitations. First, our NCDB analysis is observational which is subject to confounding, and second, the NCDB collects data from CoC-accredited hospitals, which may be limited to CoC-approved hospitals. Length of NET was not reported; therefore, we cannot determine impact of length of treatment with pCR. In our systematic review, most studies that were included had missing data with respect to tumor characteristics and length of NET that may again impact pCR outcomes. The use of observational data also introduces confounding factors, including patient 
TABLE 5 Surgical management in women with node-positive breast cancer undergoing neoadjuvant endocrine therapy

\begin{tabular}{lllll}
\hline Surgery & All patients & \multicolumn{3}{l}{ Post-neoadjuvant axillary status } \\
\cline { 3 - 5 } & & pN0 & pN+ & $p$ \\
\hline$n$ & 4580 & 663 & 3917 & \\
Primary (N, \%) & & & & \\
Lumpectomy & $1152(25.2)$ & $215(32.4)$ & $937(23.9)$ & $<0.001$ \\
Mastectomy & $3406(74.4)$ & $446(67.3)$ & $2960(75.6)$ & \\
Other/unknown & $22(0.5)$ & $2(0.3)$ & $20(0.5)$ & \\
Axilla (*2012 and later) (N, \%) & & & $<0.001$ \\
None & $80(2.4)$ & $32(6.2)$ & $48(1.7)$ & \\
Biopsy & $12(0.4)$ & $3(0.6)$ & $9(0.3)$ & \\
SLNB & $483(14.2)$ & $186(36.1)$ & $297(10.3)$ & \\
ALND & $2152(63.4)$ & $224(43.5)$ & $1928(67.0)$ & \\
SLN + ALND & $659(19.4)$ & $70(13.6)$ & $589(20.5)$ & \\
Other/unknown & $6(0.2)$ & $0(0.0)$ & $6(0.2)$ & \\
\hline
\end{tabular}

$p N O$ pathologically node-negative, $p N+$ pathologically node-positive, $N$ node, $S L N B$ sentinel lymph node biopsy, $A L N D$ axillary node dissection

demographics, genetic predisposition, and menopausal status that may bias oncologic outcomes in certain studies but cannot be accounted for in a retrospective review. The role of NET will need to be re-evaluated as additional data becomes available.

To date, the utility of NET has remained unclear, particularly in regards to the axillary response, and the usage of NET has been relatively uncommon in the United States. Recently, due to the COVID-19 pandemic; however, we have had to modify management in some breast cancer patients, particularly for those with $\mathrm{ER}^{+}$disease for whom we at least had a neoadjuvant option to employ during the delay associated with the pandemic. The results of our study may be utilized to provide more information regarding axillary downstaging in $\mathrm{ER}^{+} \mathrm{BC}$ patients during the current healthcare crisis.

\section{CONCLUSIONS}

We found that $14.5 \%$ patients who underwent NET for $\mathrm{N}^{+}$disease had a pCR in the nodal basin, which is higher than previously reported $(10 \%)$ in smaller studies. Although NET is not a common treatment option for women with $\mathrm{N}^{+}, \mathrm{ER}^{+}, \mathrm{HER}^{2-}$ breast cancer, it may be a suitable option for axillary downstaging, which is currently underutilized. Appropriate drugs and duration of NET have yet to be established, but future studies will be helpful in elucidating this topic.
AUTHOR'S CONTRIBUTION LC and KYF conceptualized and designed the project. AS and AW performed data acquisition and data analysis. AS and LC performed interpretation and drafted the initial manuscript. AS, LC, AW, KE, CC, SB, RC, and KYF assisted in critical revision of the manuscript. All authors approved the final manuscript as submitted and agree to be accountable for all aspects of the work.

DISCLOSURES No external funding was secured for this study. The authors have no financial relationships or conflicts of interest relevant to this article to disclose.

\section{REFERENCES}

1. DeSantis CE, Ma J, Gaudet MM, et al. Breast cancer statistics, 2019. CA Cancer J Clin. 2019;69(6):438-51. https://doi.org/10. 3322/caac. 21583.

2. Reinbolt RE, Mangini N, Hill JL, et al. Endocrine therapy in breast cancer: the neoadjuvant, adjuvant, and metastatic approach. Sem Oncol Nurs. 2015;31(2):146-55. https://doi.org/ 10.1016/j.soncn.2015.02.002.

3. Moy B, Goss P. Hormonal therapy of breast cancer. In: Chabner B, Lango D (eds). Cancer chemotherapy and biotherapy: principles and practice, 5th edn. Lippincott Williams \& Wilkins; 2011:651-73.

4. Mougalian SS, Soulos PR, Killelea BK, et al. Use of neoadjuvant chemotherapy for patients with stage I to III breast cancer in the United States: timing of chemotherapy in breast cancer. Cancer. 2015;121(15):2544-52. https://doi.org/10.1002/cncr.29348.

5. Clough KB, Acosta-Marín V, Nos C, et al. Rates of neoadjuvant chemotherapy and oncoplastic surgery for breast cancer surgery: a French National Survey. Ann Surg Oncol. 2015;22(11):3504-11. https://doi.org/10.1245/s10434-015-43786.

6. Vugts G, Maaskant-Braat AJG, Nieuwenhuijzen GAP, Roumen RMH, Luiten EJT, Voogd AC. Patterns of care in the administration of neo-adjuvant chemotherapy for breast cancer. a population-based study. Breast J. 2016;22(3):316-21. https://doi. org/10.1111/tbj.12568.

7. Fontein DBY, Charehbili A, Nortier JWR, et al. Efficacy of six month neoadjuvant endocrine therapy in postmenopausal, hormone receptor-positive breast cancer patients-a phase II trial. European J Cancer. 2014;50(13):2190-200. https://doi.org/10.1 016/j.ejca.2014.05.010.

8. Weiss A, Wong S, Golshan M, et al. Patterns of axillary management in stages 2 and 3 hormone receptor-positive breast cancer by initial treatment approach. Ann Surg Oncol. 2019;26(13):4326-36. https://doi.org/10.1245/s10434-019-07785 $-\mathrm{y}$.

9. Dixon JM, Renshaw L, Dixon J, Thomas J. Invasive lobular carcinoma: response to neoadjuvant letrozole therapy. Breast Cancer Res Treat. 2011;130(3):871-7. https://doi.org/10.1007/s 10549-011-1735-4.

10. Thornton MJ, Williamson HV, Westbrook KE, et al. Neoadjuvant endocrine therapy versus neoadjuvant chemotherapy in nodepositive invasive lobular carcinoma. Ann Surg Oncol. 2019;26(10):3166-77. https://doi.org/10.1245/s10434-019-07564 $-9$.

11. Winchester DP, Stewart AK, Phillips JL, Ward EE. The National Cancer Data Base: past, present, and future. Ann Surg Oncol. 2010;17(1):4-7. https://doi.org/10.1245/s10434-009-0771-3.

12. Liedtke C, Mazouni C, Hess KR, André F, Tordai A, Mejia JA, et al. Response to neoadjuvant therapy and long-term survival in patients with triple-negative breast cancer. J Clin Oncol. 2008;26:1275-1281. 
13. Fisher B, Bryant J, Wolmark N, Mamounas E, Brown A, Fisher ER, et al. Effect of preoperative chemotherapy on the outcome of women with operable breast cancer. J Clin Oncol. 1998;16(8):2672.

14. Olson JA, Jr, Budd GT, Carey LA, Harris LA, Esserman LJ, Fleming GF, et al. Improved surgical outcomes for breast cancer patients receiving neoadjuvant aromatase inhibitor therapy: results from a multicenter phase II trial. J Am Coll Surg. 2009;208:906-14.

15. Alliance for Clinical Trials in Oncology. Fulvestrant and/or anastrozole in treating postmenopausal patients with stage II-III breast cancer undergoing surgery. In: ClinicalTrials.gov. Bethesda, MD; National Library of Medicine. Available at: $\mathrm{h}$ ttps://clinicaltrials.gov/ct2/show/NCT01953588.

16. Van der Hage JA, van de Velde CJ, Julien JP, Tubiana-Hulin M, Vandervelden C, Duchateau L. Preoperative chemotherapy in primary operable breast cancer: results from the European Organization for Research and Treatment of Cancer trial 10902. $J$ Clin Oncol. 2001;19(22):4224-37.
17. Broet P, Scholl SM, de la Rochefordiere A, Fourquet A, Moreau $\mathrm{T}$, De Rycke Y, et al. Short- and long-term effects on survival in breast cancer patients treated by primary chemotherapy: an updated analysis of a randomized trial. Breast Cancer Res Treat. 1999;58(2):151-6.

18. Barcenas CH, Niu J, Zhang N, Zhang Y, Buchholz TA, Elting LS, et al. Risk of hospitalization according to chemotherapy regimen in early-stage breast cancer. J Clin Oncol. 2014;32:2010-7.

19. Kang YJ, Han W, Park S, You JY, Yi HW, Park S, et al. Outcome following sentinel lymph node biopsy-guided decisions in breast cancer patients with conversion from positive to negative axillary lymph nodes after neoadjuvant chemotherapy. Breast Cancer Res Treat. 2017;166:473-80.

Publisher's Note Springer Nature remains neutral with regard to jurisdictional claims in published maps and institutional affiliations. 